\title{
In-situ Observation of Shape Transformation and Surface Oxidation of Pd Nanocrystals
}

\author{
Aram Yoon ${ }^{1,2}$, Peng-Han Lu ${ }^{3,4}$, Zhi-Wei Shan ${ }^{3}$, Jian-Min Zuo ${ }^{1,2}$ \\ 1. Materials Science and Engineering, University of Illinois at Urbana Champaign, Urbana, IL \\ 61801, USA \\ 2. Fredrick Seitz Material Research Laboratory, University of Illinois at Urbana Champaign, \\ Urbana, IL 61801, USA \\ 3. Center for Advancing Materials Performance from the Nanoscale (CAMP-Nano), State Key \\ Laboratory for Mechanical Behavior of Materials, Xi' an Jiaotong University, Xi'an 710049, \\ China \\ 4. Ernst Ruska-Centre for Microscopy and Spectroscopy with Electrons and Peter Grünberg \\ Institute, Forschungszentrum Jülich GmbH, 52425 Jülich, Germany
}

Palladium nanoparticles (NPs) are metal catalysts employed in a wide range of applications, such as automotive catalytic converters for oxidation and reduction of pollutant gases $\left(\mathrm{CO}\right.$ or $\left.\mathrm{NO}_{\mathrm{x}}\right)$. The superior catalytic properties of palladium NPs have motivated considerable research to study their structure and property relationships. The atomic structure of palladium NP surfaces is of particular interest, since catalytic reaction is believed to occur at the surface of the NPs. The surfaces of the Pd metal were previously studied under oxygen environment using standard surface science techniques. Several oxide structures on 2D Pd bulk surfaces have been found under various redox conditions. However, we know little about the nanoparticle surfaces and it is not yet known which nanocrystal surface structure forms under the reactive conditions.

The surface of a nanocrystal differs from the 2D bulk surface because of their smaller size and 3D shape. Nanocrystals consist of facets with different crystal planes and contain many corners and edges. The different crystal planes of the facets have different reactivities for oxygen absorption and oxide formation. Consequently, the 3D shape of the nanocrystal may undergo anisotropic changes during the reaction. On the other hand, the catalytic activity of nanocrystals depends sensitively on the number of sites at the corners and edges [1]. The corners and edges have been suggested to be chemically active because of their lower coordination, and previous studies have demonstrated that their presence induces surface reconstruction [2]. These differences make it important to directly study the surface structure of 3D nanocrystals in-situ and under controlled environment.

Here, we observe the shape changes of Pd nanocrystals and their surface structure using an environmental transmission electron microscope (ETEM). ETEM enables us to obtain real-time images of the palladium nanoparticles during reactions with oxygen gas at elevated temperatures. The palladium nanocrystals were prepared on an oxide powder support using an e-beam evaporator and annealed at $300{ }^{\circ} \mathrm{C}$ to form equilibrium shapes before oxidation. Oxidation was done inside the ETEM at $300-400{ }^{\circ} \mathrm{C}$ with $2 \times 10^{-5}$ mbar oxygen pressure.

Palladium nanocrystals showed reversible shape changes (Figure 1), as expected from the previous studies $[3,4]$. The equilibrium shapes of nanocrystals were truncated octahedra in vacuum, but the nanocrystal became rounded in shape at higher oxygen pressures. The sharp edges of the truncated octahedron, representing the intersection of $\{111\}$ facets, were smoothed 
as new crystal planes were exposed during oxidation. These new crystal planes were identified as $\{110\}$ planes. The $\{110\}$ planes did not exist before oxidation due to their higher surface energy compared to the $\{111\}$ and $\{100\}$ planes. Consequently, the appearance of them at higher oxygen pressures was evidence for a surface energy change among the crystal planes. We also found that the shape change was reversible, i.e. the Pd nanocrystal went back to its original sharp shape after the oxygen gas was purged.

In addition to the shape changes, we observed surface modifications of the palladium nanocrystals under oxygen atmosphere. The complex oxide structure was formed on the surface of the nanocrystal and did not correspond to any stable phase of the bulk oxide or the surface oxide found at the 2D bulk surface. This new oxide phase is suspected to be a kinetically metastable phase, associated with either strain or oxygen deficiency (suboxide). Further investigation of this new phase and its thermodynamic validation will be discussed in the paper. We will also discuss the relation between the surface oxides and the shape changes of Pd nanocrystals.

\section{References}

[1] M. Cargnello, V. V. T. Doan-Nguyen, T. R. Gordon, R. E. Diaz, E. A. Stach, R. J. Gorte, P. Fornasiero, and C. B. Murray, Science 341 (2013), pp.771-773.

[2] W. J. Huang, R. Sun, J. Tao, L. D. Menard, R. G. Nuzzo, and J. M. Zuo, Nature Materials 7 (2008), pp. $308-313$

[3] F. Mittendorfer, N. Seriani, O. Dubay, and G. Kresse, Phys. Rev. B 76 (2007), p. 233413.

[4] P. Nolte, A. Stierle, N. Kasper, N. Y. J. Phillipp, N. Jeutter, and H. Dosch, Nano Lett. 11 (2011), pp.4697-4700.

[5] The authors acknowledge funding from the National Science Foundation, USA, and Kwanjeong Educational Foundation, Korea. The ETEM is supported by DMR 12-29454. JMZ and AY are partially supported by DMR 14-10596, and AY is additionally supported by Kwanjeong Educational Foundation.
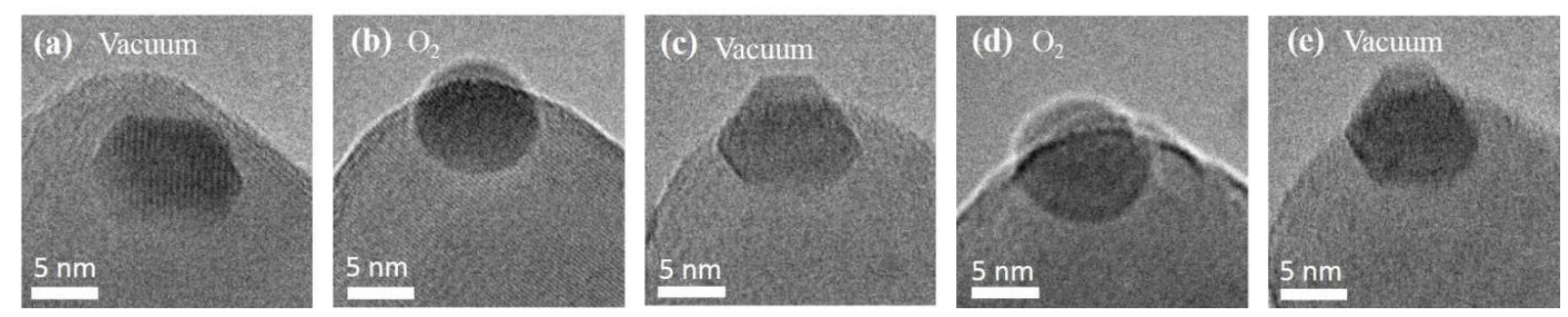

Figure 1. The shapes of a $\mathrm{Pd}$ nanocrystal observed on the $\mathrm{TiO}_{2}$ nanoparticle support. The shape changed responding to the gas pressure changes. The truncated octahedral shape observed under vacuum became rounded after introducing oxygen. The shape change was reversible during the purging/introduction of oxygen gas. The vacuum here is the TEM base pressure of $10^{-7} \mathrm{mbar}$ in (a), (c) and (e), and $\mathrm{O}_{2}$ in (b) and (d) is at the oxygen pressure of $2 \times 10^{-5}$ mbar after $\mathrm{O}_{2}$ gas injection. 